\title{
Wind Turbine Gearbox fault early warning based on NEST
}

\author{
Xiao Yan ${ }^{1, a}$, Changliang Liü $^{2, b}$ \\ ${ }^{1}$ School of Control and Computer Engineering,North China Electric Power University,Baoding \\ 071000, China \\ ${ }^{2}$ State Key Laboratory Of Alternate Electrical Power System With Renewable Energy \\ Sources(NCEPU),Beijing 102206,China \\ a e-mail: stellar1207@126.com, be-mail: changliang_liu@163.com
}

Keywords: Fault early warning; NEST; Grey Relational Analysis ; Residual characteristic analysis

\begin{abstract}
The condition detection of gearbox is of great significance to improve the operation level and reduce the maintenance cost of the wind turbine. Based on the nonlinear state estimation (NSET) method, the temperature model of the gear box bearing is set up to estimate the states of gear box and the estimated value of the gearbox bearing temperature is obtained. Having obtained the residual error of the estimated value and the actual value of the gearbox bearing temperature, the residual distribution characteristics are analyzed by sliding window error statistics method. The residual distribution characteristic of the model will be changed when the working condition of the gearbox is abnormal, and when the residual mean or standard deviation exceeds the set threshold, the warning is given.
\end{abstract}

\section{Introduction}

The gearox is one of the important components of wind turbine, which manufacturing technology has been more mature and reliable. Due to frequent alternating load, impact load, and the strong coupling among parts, the gearbox composed of gear, bearing and other wearing parts has become wind turbine functional system with the highest failure rate. Therefore, in order to ensure the safe and stable operation of the wind turbine and reduce maintenance expenses, the real-time monitoring of is very necessary.

[1]used multiple layer neural network algorithm for gear box and generator modeling and set threshold according to the temperature. The he diagnosis result of different parts could be comprehensive analyzed for the fault prediction with Multi-agent methods. At the same time the author pointed out that the algorithm accuracy of neural network was affected by many factors, such as network structure, training algorithm selection, object modeling and learning sample. A fault early warning method of wind turbine based on least squares support vector machine (LS-SVM) was proposed in[2]. The feasibility of this method was verified with the monitoring data of wind turbine operation. Although some achievements have been achived by these methods, there are still some limitations.

Nonlinear state estimate technique (NSET) was applied to construct the normal operating model of the wind tower vibration to analyze the operation state of wind turbine in [3]. Through two validation examples it showed that the NSET modeling method for tower vibration had the merits of simplicity, direct physical interpretation and high accuracy.

This paper uses a method based on gearbox temperature trend analysis based on the Nonlinear State Estimate Technique (NSET) to monitor the condition of the gearbox.

\section{Nonlinear state estimate technique model construction}

\section{Modeling parameters selection}

The key to the NSET algorithm for gearbox temperature modeling is to determine the variable set closely related to the temperature e change of the gear box bearing reasonably, that is to determine the history matrix structure and the operating parameters of the observation vector. 
Through the qualitative analysis of the gear box bearing temperature in[4] and [5], this paper firstly selected as much as possible types of the variable factors which affect the output greatly. Selected model variables were the following nine wind power unit operation parameters: power, wind speed, rotational speed of the generator, wind direction angle, bearing temperature of gearbox, gearbox oil temperature, room temperature, ambient temperature, the generator torque.

When there are many variables and make them all the inputs of the model, it will obviously increase the complexity of the model, calculate the running time and affect model accuracy. So use Grey Relational Analysis (GRA) to solve this problem. After the degree of correlation is obtained, the correlation degree between the comparison sequence and the reference sequence is from large to small and the greater the degree of correlation, the more consistent with the changing trend of the sequence and reference sequence and the influence of the comparison sequence on the reference sequence is more significant. Through the calculation of GRA, we can deal with a large number of input variables and we can select the variables more comprehensive and extensive rather than going through a special subjective screening, which enhance the adaptability of the model.

Table.1. The correlation degree between the parameters and the temperature

\begin{tabular}{|c|c|c|c|c|c|c|c|c|}
\hline parameters & $\mathrm{R}_{1}$ & $\mathrm{R}_{2}$ & $\mathrm{R}_{3}$ & $\mathrm{R}_{4}$ & $\mathrm{R}_{5}$ & $\mathrm{R}_{6}$ & $\mathrm{R}_{7}$ & $\mathrm{R}_{8}$ \\
\hline correlation degree & 0.86 & 0.96 & 0.57 & 0.92 & 0.99 & 0.97 & 0.96 & 0.46 \\
\hline
\end{tabular}

In the table above, $\mathrm{R}_{1}$ is grey relational degree of wind power and the gearbox bearing temperature, $R_{2}$ is grey relational degree of wind speed and the gearbox bearing temperature, $R_{3}$ is grey relational degree of rotational speed and the gearbox bearing temperature, $R_{4}$ is grey relational degree of wind angle and the gearbox bearing temperature, $R_{5}$ is grey relational degree of tank temperature of gear box and the gearbox bearing temperature, $\mathrm{R}_{6}$ is grey relational degree of engine room temperature and the gearbox bearing temperature, $R_{7}$ is grey relational degree of ambient temperature and the gearbox bearing temperature, $R_{8}$ is grey relational degree of real time torque and the gearbox bearing temperature. The calculation result shows that the wind speed, wind angle, tank temperature of gearbox, engine room temperature and ambient temperature have a large degree of correlation with gearbox bearing temperature, so we choose them as the NSET model parameter variable.

\section{NSET model algorithm principle}

Nonlinear state estimation technique (NEST) is a kind of data-driven modeling method proposed by singer [J] and at present it has successful applications in nuclear power plant sensor calibration ${ }^{[6]}$, the excitation energy transfer ${ }^{[7]}$, the fault diagnosis ${ }^{[8]}$.

There are n correlative variables in variable set of a process or device. At time i, the observation of the variables can be written as an observation vector:

$$
X(i)=\left[\begin{array}{lll}
x_{1} X & \cdots & x_{n}
\end{array}\right]^{T}
$$

The structure of the process memory matrix D is the first step in the NSET modeling. In the course of the operation of the process or equipment, under different operating conditions (such as low load, high load, etc.) to collect m historical observation vectors to form the memory matrix:

$$
\begin{aligned}
& D=\left[\begin{array}{llll}
X(1) & X(2) & \cdots & X(m)
\end{array}\right]= \\
& {\left[\begin{array}{cccc}
x_{1}(1) & x_{1}(2) & \cdots & x_{1}(m) \\
x_{2}(1) & x_{2}(2) & \cdots & x_{2}(m) \\
\vdots & \vdots & & \vdots \\
x_{n}(1) & x_{n}(2) & \cdots & x_{n}(m)
\end{array}\right]_{n \times m}}
\end{aligned}
$$

Each column of the observation vector in the process memory matrix represents a normal operating state of the device. With proper selection of the $\mathrm{m}$ historical observation vectors, the subset space spanned by the memory matrix D can represent the whole normal working space of the process or device. Therefore, the construction of the process memorizing matrix is the process of learning and memory of the normal operation of the process or equipment.

The input of the NSET model is a new observation vector Xobs of the process or equipment at a certain time and the output of the model is the estimated value Xest of the input vector. For any 
input observation vector Xobs, NSET generates a m dimension of the weight vector $\mathrm{W}$ :

$$
W=\left[\begin{array}{lll}
W_{1} W & \cdots & W_{m}
\end{array}\right]^{T}
$$

with:

$$
X_{\text {est }}=w_{1} X(1)+w_{2} X(2)+\cdots+w_{m} X(m)
$$

Weight is the measure of the similarity between the state estimation and the memory matrix, which can be calculated in the following way. The residual of the state estimation matrix and observation matrix is $\varepsilon$ and seek its minimum value:

$$
\begin{aligned}
& \varepsilon=X_{o b s}-X_{e s t} \\
& \min \varepsilon=\min \left(\left|X_{o b s}-D W\right|\right)
\end{aligned}
$$

Sum of squares of residuals:

$$
\min \varepsilon^{2}=\sum_{i=1}^{n}\left(X_{o b s}(i)-\sum_{j=1}^{m} W_{j} D_{i j}\right)^{2}
$$

In order to minimize the residual, we need to obtain the minimum value of $\varepsilon^{2}$. Let the partial difference of $\varepsilon^{2}$ with respect to be zero as follows:

$$
\frac{\partial \varepsilon^{2}}{\partial W_{k}}=-2 \sum_{i=1}^{n}\left(X_{o b s}(i)-\sum_{j=1}^{m} W_{j} D_{i j}\right) D_{i k}=0
$$

That is:

$$
\sum_{i=1}^{n} X_{o b s}(i) D_{i k}=\sum_{j=1}^{m}\left(\sum_{j=1}^{n} D_{i j} D_{i k}\right)_{W_{j}}(k=1 \cdots m)
$$

Lastly we can obtain the weight vector $W$ as:

$$
W=\left(D^{T} \cdot D\right)^{-1} \cdot\left(D^{T} \cdot X_{\text {obs }}\right)
$$

There is a certain correlation between the state data of most systems, the correlation between the data can make the matrix in formula (10) irreversible, which limits the obtain of the weight. On this occasion, euclidean distance is used to reflect the relationship between the two vectors in order to make the nonlinear state estimation method more intuitive in NSET method, which use similarity based on the similarity principle. Characterize its weight and solve the irreversibility of the matrix caused by the correlation of the data by calculating the degree of similarity between the data states. We apply the similarity operator instead of the dot product and (10) can be written as:

$$
W=\left(D^{T} \otimes D\right)^{-1} \cdot\left(D^{T} \otimes X_{o b s}\right)
$$

According to the generalized weights, the relationship between the current state estimation matrix and the observation matrix of the system is as follows:

$$
X_{e s t}=D \cdot\left(D^{T} \otimes D\right)^{-1} \cdot\left(D^{T} \otimes X_{o b s}\right)
$$

Sliding window residual method

The mean value of the residual sequence can reflect the change of its size and distribution. By using the method of sliding window error statistics, the variation of the residual distribution characteristics can be analyzed in real time. Choosing the width of the $\mathrm{N}$ sliding window reasonably can not only reflect the continuous change of the statistical characteristics of the residuals in a timely manner but also eliminate the influence of random factors, which finally improves the reliability of the condition monitoring of gear box and reduces the probability of false alarm. Temperature residual of gear box bearing is defined as:

$$
\varepsilon=X_{o b s}-X_{e s t}
$$

For a certain period of time, the gearbox bearing temperature residual series of NSET model is:

$\varepsilon_{G T}=\left[\begin{array}{lllll}\varepsilon_{1} & \varepsilon_{2} & \cdots & \varepsilon_{N} & \cdots\end{array}\right]$

For the above residual sequence, the sliding window with the width of $\mathrm{N}$ is taken to calculate the mean value and standard deviation of the continuous $\mathrm{N}$ residual in this window: 


$$
\begin{gathered}
\bar{X}_{\varepsilon}=\frac{1}{N} \sum_{i=1}^{N} \varepsilon_{i} \\
S_{c}=\sqrt{\frac{1}{N-1} \sum_{i=1}^{N}\left(\varepsilon_{i}-\bar{X}_{\varepsilon}\right)^{2}}
\end{gathered}
$$

\section{The Validation of the Model}

\section{The Validation of gearbox Bearing Temperature NSET Model}

The rated power of the wind turbine researched in this paper is $1.5 \mathrm{MW}$, the cut in wind speed is $3 \mathrm{~m} / \mathrm{s}$ and the rated wind speed is $10.8 \mathrm{~m} / \mathrm{s}$. The wind turbine once recorded 69 operating parameters such as the main information time, power, wind speed, generator speed, wind direction, gear box bearing temperature, gear box temperature, engine room temperature, ambient temperature, real-time torque, vibration acceleration on both sides of the engine room, etc. According to the second section, this paper selected the following six wind turbine operating parameters as the NSET model variables: wind speed, wind direction angle, gear box bearing temperature, gear box tank temperature, engine room temperature, ambient temperature.

The running data of the wind turbine in SCADA system is huge, and the data contains the data points of the abnormal operation of the unit, which will affect the reliability of the results. When constructing the memory matrix, it is necessary to remove the data from the normal operation of the wind turbine.

This paper selects appropriate number of data according to a certain time and ensures the selected data including wind turbine operation conditions. At the same time exclude those points whose output power is zero or less than zero, whose wind speed is less than $3 \mathrm{~m} / \mathrm{s}$ or whose beyond $25 \mathrm{~m} / \mathrm{s}$, which considered as points under abnormal operating conditions.

Finally, we select the 2254 sets of data form the SCADA system structure memory matrix D, and the 800 sets of data as the test samples of the NSET model. Validation results are shown in figure 1.

From the Fig 1 we can see that the NSET model of gearbox bearing temperature has a high modeling accuracy for the dynamic characteristics of the gearbox in normal operation, and the curve can track the change of gearbox temperature. What's more, the residual is very small that we can accurately get the gear box temperature estimated residual value.

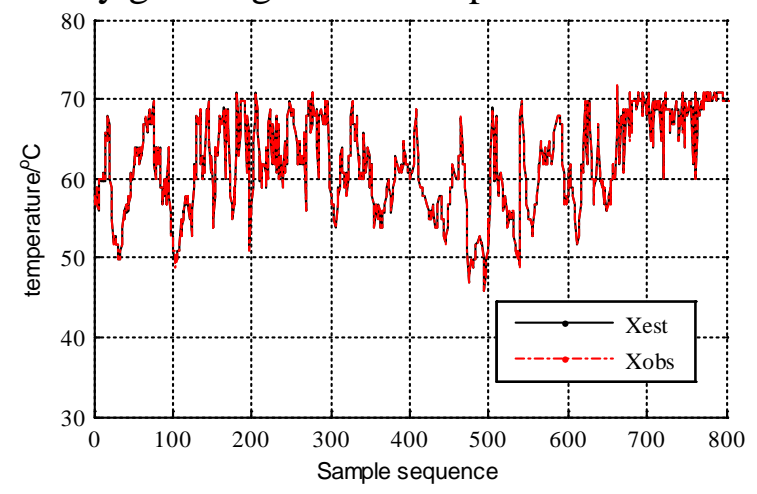

(a) Estimation result of the model

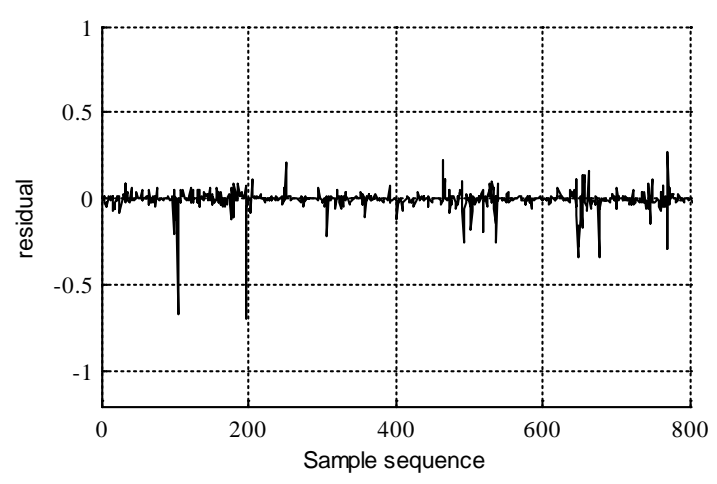

(b) Residual distribution

Fig.1. simulation result of NEST

\section{Temperature trend estimation of gearbox working abnormal}

In order to test the validity of the above model, the temperature of the gear box bearing is increased artificially to simulate the situation of the gearbox bearing temperature rising when the gear box is in trouble.

To detect the faults of gearbox based on the variation of the residual statistic characteristic, the threshold value of the residual mean and the standard deviation should be determined, denoted as Ev and Sv respectively. When the residual statistic characteristic of the model is beyond the threshold value, it can send out the hidden trouble alarm to prompt the operator to pay attention to the running state of the gearbox and take the corresponding treatment measures. 
In the last section, the results of NSET model validation are used as the health indicators of the gear box under normal conditions. In this paper, the sliding window width $\mathrm{N}=90$. Thus, we set the residual mean threshold value $\mathrm{Ev}=0.098$, and the residual standard deviation threshold value $\mathrm{Sv}=0.05$.

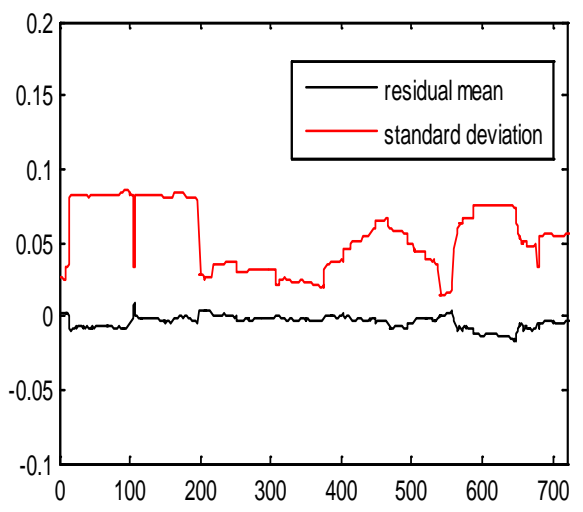

Fig.2.Residual distribution characteristics

From point 450, a manual drift with step 0.008 is added to the 800 normal operating data validating sequence to simulate the fault condition of the gearbox. Simulation of NSET model is shown in Fig3. (a) is a comparison chart of the observation vector and the estimated vector and (b) is he residual between the two vectors.

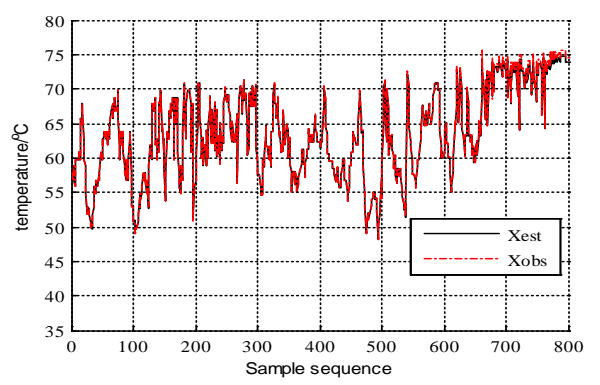

(a) Estimation result of the model

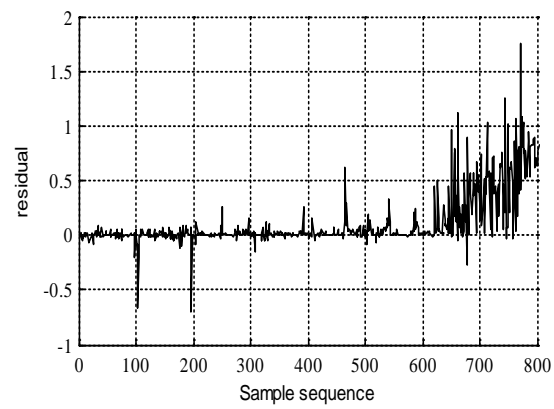

(b) Residual distribution

Fig.3. Residual distribution of Simulating sequence after adding manual drift

Then the residual is processed by the sliding window. Here the sliding window width $\mathrm{N}$ is set 90 , and the statistical properties of model prediction is shown in Fig 4. From Fig.4, we can see that the residual mean and standard deviation begin to increase after the manual drift added from point around 480. The upper limit of the confidence interval beyond the preset mean threshold in the 550st sliding window. It is thus clear that when the gear box has hidden trouble, the temperature variation is abnormal, the dynamic characteristic of the gear box is changed so the input observation vector is deviated from the normal working space that the model can't construct its exact estimated value through the combination of historical observation vectors in the process memory matrix $\mathrm{D}$.

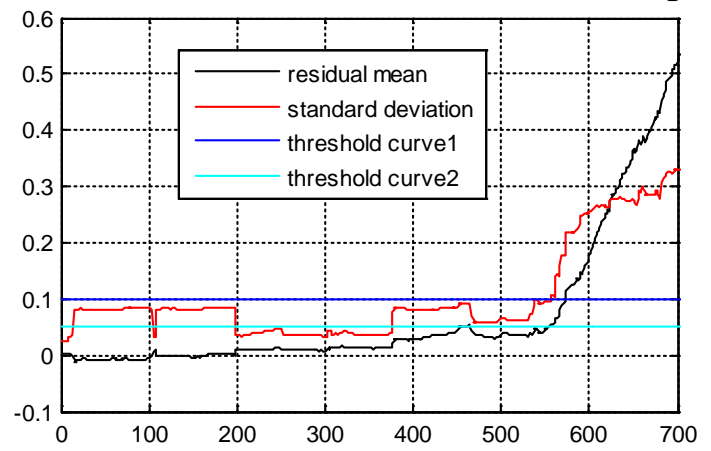

Fig.4.Residual distribution characteristics after adding manual drift 


\section{Conclusion}

In this paper, the NSET method is used to set up the gearbox bearing temperature prediction model by using the SCADA system data of the wind turbine. NSET modeling algorithm is simple with clear physical meaning which is very suitable for modeling complex stochastic processes. Grey relational analysis is used to overcome the subjectivity when selecting the appropriate variable. Then use the sliding window method to analyze the prediction residual of the temperature model of the gearbox bearing, and analyze the variation of the distribution characteristic of the residual distribution, so as to give the indication of the potential fault signal. Simulation results show that the temperature model of the gearbox bearing of the wind turbine based on the NSET method can be used to monitor the operation status of the gearbox in real time, carry out real-time fault diagnosis of the running status of the gearbox and give a warning in advance so as to avoid or reduce the damage to the equipment, lower the maintenance costs and improving the safety and economy of the operation of the wind turbine.

\section{Acknowledgement}

This project was supported by State Key Laboratory of Alternate Electrical Power System with Renewable Energy Sources. Thanks a lot.

\section{References}

[1] ZAHER A,MCARTHURS DJ,INFIELDDG.Online wind turbine fault detection through automated SCADA data analysis[J].WindEnergy,2009,12(6):574-593.

[2] XU JunLong, LI Zheng. Fault early warning of wind turbine generator based on support vector machine[J]. Industrial Control Computer, 2013,08:54-56.

[3] GUO Peng, XU Ming, BAI Nan, et al. Wind Turbine Tower Vibration Modeling and Monitoring Driven by SCADA Data[J.] Proceedings of the CSEE, 2013,05:128-135+20..

[4] SUN Jian-ping, ZHU Wen, ZHAI Yong-jie, GE Jian-hong. Wind turbine gearbox prognostic simulation research based on MSET method[J]. Journal of System Simulation, 2013,12:3009-3014..

[5] GUO Peng, DAVID Infield, YANG Xiyun. Wind turbine gearbox condition monitoring using temperature trend analysis[J].Proceedings of the CSEE, 2011,32:129-136.

[6] Bockhorst F K, Gross K C, Herzog J P, et al. MSET Modeling of Crystal River-3 Venturi Flow Meters [C]// Proceedings of International Conference on Nuclear Engineering, USA: IEEE, 1998: 128-134.

[7] Jingfang Guo,Yamei Yang,Xiaoli Hu,Yuanfang Li. Spectrofluorimetric analysis of captopril based on its obstruction effect of the nanomaterial surface energy transfer between acridine orange and gold nanoparticles[J]. Science China(Chemistry),2015,05:885-891.

[8] REN Mengwei,JIAO Songming.Coal milling system fault diagnosis based on improved NEST[J]. Thermal Power Generation, 2015,12:87-92+97.

[9] Peng Guo School of Control and Computer Engineering, North China Electric Power University, Beijing 102206. Wind Turbine Generator Bearing Condition Monitoring with NEST Method[A]..

[10]SUN Jian-ping, ZHU Wen, ZHAI Yong-jie, GE Jian-hong. Wind Turbine Gearbox Prognostic Simulation Research Based on MSET Method[J]. Journal of System Simulation, 2013,12:3009-3014. 